\title{
EL AISLAMIENTO TERMICO DE LOS SISTEMAS DRAGADOS-PLASTBAU I y II
}

M. Domínguez, Instituto del Frio/C.S.I.C.

J. A. Carrasco, Instituto del Frio/C.S.I.C.

J. A. Comas, Dragados y Construcciones, S. A.

El estudio del comportamiento térmico de los muros DRAGADOS-PLASTBAU (D-P) se ha llevado a cabo en el Instituto del Frio. C.S.I.C.

En este trabajo se dan a conocer los resultados del estudio térmico tanto en régimen estacionario, para verano e invierno, como en régimen variable para diversas perturbaciones periódicas sinusoidales e incluso para la curva típica de temperatura del mes de enero en Madrid.

Se describen asimismo las técnicas de ensayo utilizadas y los principios en que se han basado los cálculos teóricos.

Los muros D-P I y II analizados se han representado esquemáticamente en las figuras 1 y 2 . La densidad nominal del poliestireno expandido empleado fue de $15-17 \mathrm{~kg} / \mathrm{m}^{3}$ y el espesor de las cáscaras de microhormigón fueron comprobadas una vez finalizados los ensayos.

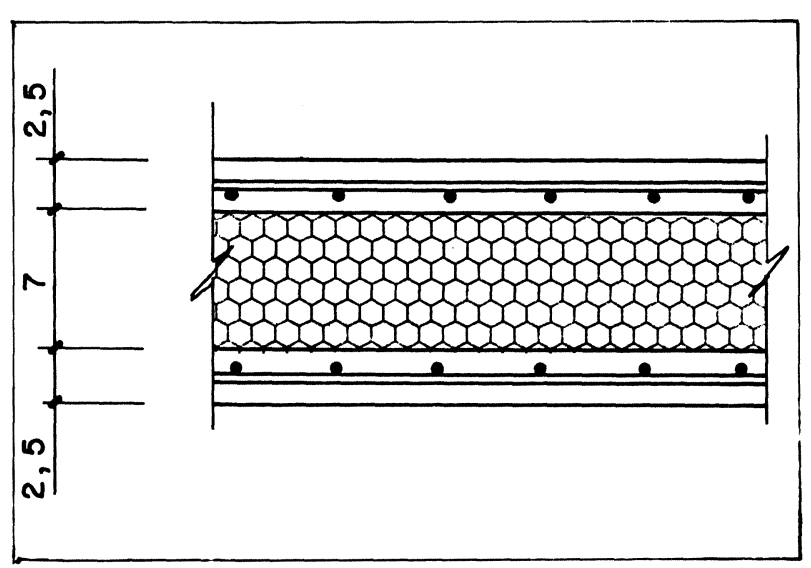

Fig. 1. - Dragados-Plastbau I.



Fig. 2. - Dragados-Plastbau II.

\section{DESCRIPCION DEL LABORATORIO}

La cámara de ensayos del Instituto (figura 3) está formada por dos recintos, cámara fría y ca-

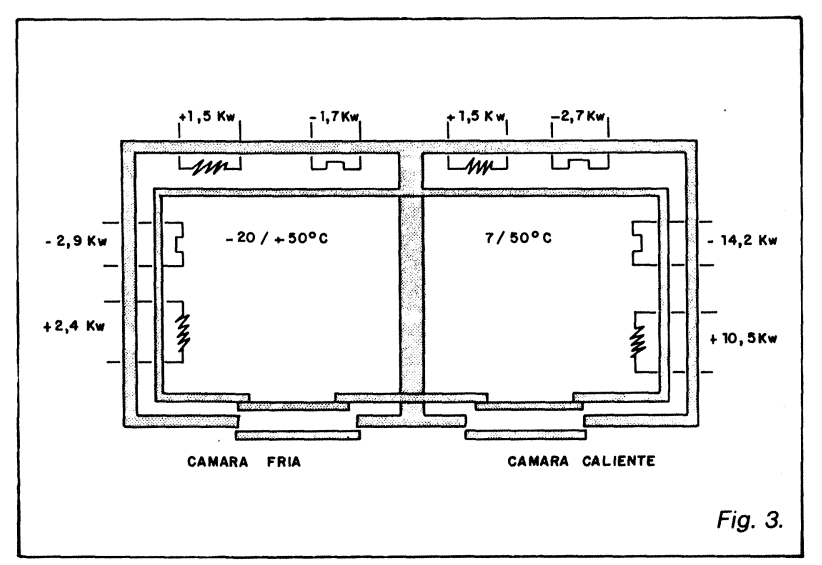


liente, separados por el muro a ensayar. Cada uno de estos recintos dispone, tanto en la parte central como en la periférica, de instalaciones de calor y frío, así como de una circulación de aire adecuada, que permiten obtener temperaturas entre $-200 \mathrm{C}$ y $50 \circ \mathrm{C}$. Esta parte del laboratorio ha sido ampliamente descrita en otras publicaciones (1), (2) por lo que no nos detendremos más en su descripción.

Lo más importante de este laboratorio es la posibilidad de conseguir una amplia gama de variaciones de temperatura.

Esto se realiza programando la función temperatura-tiempo en un ordenador y utilizando el valor correspondiente a cada instante como punto de referencia del sistema de control automático de la cámara, con lo que se consigue variar de forma precisa la temperatura en ella y seguir la función programada.

De esta forma hemos obtenido variaciones de temperatura tipo seno con distinto periodo, tipo escalón y pico o la de un día típico de cálculo.

El laboratorio está dotado de un sistema de adquisición de datos con capacidad para 256 sondas que pueden ser de termopares o captadores con salida en milivoltios o voltios, conectado a un ordenador HP 9826.
Las temperaturas se han medido con termopares de cobre-constantán de $0,5 \mathrm{~mm}$ de diámetro y los flujos térmicos con fluxímetros de varias dimensiones, recogiéndose las medidas en disco para su ulterior procesado y discusión.

\section{ENSAYOS EN REGIMEN ESTACIONARIO}

En los muros construidos, representativos de este sistema, se diferenciaron zonas de conectores, separados a $100 \times 300 \mathrm{~mm}$ y $300 \times 300$ $\mathrm{mm}$, zonas de huecos del poliestireno rellenos de hormigón y vacios, asi como zonas de unión de paneles.

En cada uno de los habitáculos separados por el muro a ensayar se reprodujeron las condiciones equivalentes al interior y exterior de la vivienda.

Las condiciones de ensayo en régimen estacionario fueron:

\section{Dragados-Plastbau I}

Ambiente interno ........... $220 \mathrm{C}$

Ambiente externo............. $30 \mathrm{C}$ y $45^{\circ} \mathrm{C}$

Dragados-Plastbau II

Ambiente interno .......... 250 C

Ambiente externo.............. $4,5 \circ \mathrm{C}$ y $440 \mathrm{C}$

TABLA 1

DRAGADOS-PLASTBAU I

\begin{tabular}{|c|c|c|c|c|c|c|}
\hline Zona & \multicolumn{4}{|c|}{ Magnitudes } & $\begin{array}{l}\text { Valores } \\
\text { Ensayo } 1\end{array}$ & $\begin{array}{l}\text { Valores } \\
\text { Ensayo 2 }\end{array}$ \\
\hline $\begin{array}{c}\text { Conectores } \\
100 \times 300 \mathrm{~mm}\end{array}$ & $\begin{array}{l}\text { T. Aire } \\
\text { T. muro } \\
\text { T. muro } \\
\text { T. aire } \\
\text { Flujo calor } \\
\text { C. Global real } \\
\text { C. T. muro } \\
\text { C. Global normalizado }\end{array}$ & $\begin{array}{l}T_{e 1} \\
T_{s 1} \\
T_{s 2} \\
T_{i 2} \\
\varnothing \\
U_{R} \\
C_{M} \\
U\end{array}$ & $\begin{array}{l}\circ \mathrm{C} \\
\circ \mathrm{C} \\
\circ \mathrm{C} \\
\circ \mathrm{C} \\
\mathrm{Kcal} / \mathrm{h} \cdot \mathrm{m}^{2} \\
\mathrm{Kcal} / \mathrm{h} \cdot \mathrm{m}^{2} \\
\mathrm{Kcal} / \mathrm{h} \cdot \mathrm{m}^{2} \\
\mathrm{Kcal} / \mathrm{h} \cdot \mathrm{m}^{2}\end{array}$ & $\begin{array}{l}\circ \mathrm{C} \\
\circ \mathrm{C} \\
\circ \mathrm{C} \\
\end{array}$ & $\begin{array}{c}3.0 \\
3.5 \\
21.3 \\
21.9 \\
6.84 \\
0.363 \\
0.383 \\
0.357 \\
\end{array}$ & $\begin{array}{l}21.0 \\
22.0 \\
45.6 \\
46.0 \\
11.24 \\
0.454 \\
0.476 \\
0.436 \\
\end{array}$ \\
\hline $\begin{array}{c}\text { Conectores } \\
300 \times 300 \mathrm{~mm}\end{array}$ & $\begin{array}{l}\text { T. aire } \\
\text { T. muro } \\
\text { T. muro } \\
\text { T. aire } \\
\text { Flujo calor } \varnothing \\
\text { C. Global real } \\
\text { C. T. muro } \\
\text { C. Global normalizado }\end{array}$ & $\begin{array}{l}\mathrm{T}_{\mathrm{e} 1} \\
\mathrm{~T}_{\mathrm{s} 1} \\
\mathrm{~T}_{\mathrm{s} 2} \\
\mathrm{~T}_{\mathrm{i} 2} \\
\varnothing \\
\mathrm{U}_{\mathrm{R}} \\
\mathrm{C}_{\mathrm{M}} \\
\mathrm{U} \\
\end{array}$ & $\begin{array}{l}\circ \mathrm{C} \\
\circ \mathrm{C} \\
\circ \mathrm{C} \\
\circ \mathrm{C} \\
\mathrm{Kcal} / \mathrm{h} \cdot \mathrm{m}^{2} \\
\mathrm{Kcal} / \mathrm{h} \cdot \mathrm{m}^{2} \\
\mathrm{Kcal} / \mathrm{h} \cdot \mathrm{m}^{2} \\
\mathrm{Kcal} / \mathrm{h} \cdot \mathrm{m}^{2}\end{array}$ & $\begin{array}{l}\circ \mathrm{C} \\
\circ \mathrm{C} \\
\circ \mathrm{C} \\
\end{array}$ & $\begin{array}{c}3.0 \\
3.5 \\
21.9 \\
22.4 \\
6.66 \\
0.344 \\
0.362 \\
0.338 \\
\end{array}$ & $\begin{array}{c}21.4 \\
22.3 \\
44.7 \\
45.2 \\
10.99 \\
0.461 \\
0.489 \\
0.447 \\
\end{array}$ \\
\hline $\begin{array}{l}\text { Conectores } \\
300 \times 300 \mathrm{~mm} \\
\text { en junta }\end{array}$ & $\begin{array}{l}\text { T. aire } \\
\text { T. muro } \\
\text { T. muro } \\
\text { T. aire } \\
\text { Flujo calor } \\
\text { C. Global real } \\
\text { C. T. muro } \\
\text { C. Global normalizado }\end{array}$ & $\begin{array}{l}\mathrm{T}_{\mathrm{e} 1} \\
\mathrm{~T}_{\mathrm{s} 1} \\
\mathrm{~T}_{\mathrm{s} 2} \\
\mathrm{~T}_{\mathrm{i} 2} \\
\varnothing \\
\mathrm{U}_{\mathrm{R}} \\
\mathrm{C}_{\mathrm{M}} \\
U\end{array}$ & $\begin{array}{l}\circ \mathrm{C} \\
\circ \mathrm{C} \\
\circ \mathrm{C} \\
\circ \mathrm{C} \\
\mathrm{Kcal} / \mathrm{h} \cdot \mathrm{m}^{2} \\
\mathrm{Kcal} / \mathrm{h} \cdot \mathrm{m}^{2} \\
\mathrm{Kcal} / \mathrm{h} \cdot \mathrm{m}^{2} \\
\mathrm{Kcal} / \mathrm{h} .^{2}\end{array}$ & $\begin{array}{l}\circ \mathrm{C} \\
\circ \mathrm{C} \\
\circ \mathrm{C}\end{array}$ & $\begin{array}{c}3.0 \\
3.6 \\
22.0 \\
22.4 \\
7.45 \\
0.383 \\
0.406 \\
0.376\end{array}$ & $\begin{array}{l}21.7 \\
22.3 \\
44.5 \\
45.1 \\
11.45 \\
0.490 \\
0.516 \\
0.470\end{array}$ \\
\hline
\end{tabular}


TABLA 2

DRAGADOS-PLASTBAU ॥

\begin{tabular}{|c|c|c|c|c|c|c|}
\hline Zona & \multicolumn{4}{|c|}{ Magnitudes } & $\begin{array}{l}\text { Valores } \\
\text { Ensayo } 1\end{array}$ & $\begin{array}{l}\text { Valores } \\
\text { Ensayo } 2\end{array}$ \\
\hline $\begin{array}{c}\text { Conectores } \\
100 \times 300 \mathrm{~mm} \\
\text { Ilenos los } \\
\text { huecos de hormigón }\end{array}$ & $\begin{array}{l}\text { T. aire } \\
\text { T. muro } \\
\text { T. muro } \\
\text { T. aire } \\
\text { Flujo calor } \\
\text { C. Global real } \\
\text { C. T. muro } \\
\text { C. Global normalizado }\end{array}$ & $\begin{array}{l}\mathrm{T}_{\mathrm{e} 1} \\
\mathrm{~T}_{\mathrm{s} 1} \\
\mathrm{~T}_{\mathrm{s} 2} \\
\mathrm{~T}_{\mathrm{i} 2} \\
\varnothing \\
\mathrm{U}_{\mathrm{R}} \\
\mathrm{C}_{\mathrm{M}} \\
\mathrm{U}\end{array}$ & $\begin{array}{l}\circ \mathrm{C} \\
\circ \mathrm{C} \\
\circ \mathrm{C} \\
\circ \mathrm{C} \\
\mathrm{Kcal} / \mathrm{h} \cdot \mathrm{m}^{2} \\
\mathrm{Kcal} / \mathrm{h} \cdot \mathrm{m}^{2} \\
\mathrm{Kcal} / \mathrm{h} \cdot \mathrm{m}^{2} \\
\mathrm{Kcal} / \mathrm{h} \cdot \mathrm{m}^{2} \\
\end{array}$ & $\begin{array}{l}\circ \mathrm{C} \\
\circ \mathrm{C} \\
\circ \mathrm{C} \\
\end{array}$ & $\begin{array}{c}4.5 \\
5.2 \\
24.9 \\
25.1 \\
6.00 \\
0.292 \\
0.305 \\
0.288 \\
\end{array}$ & $\begin{array}{l}25.3 \\
25.7 \\
44.0 \\
44.2 \\
6.99 \\
0.377 \\
0.382 \\
0.356 \\
\end{array}$ \\
\hline $\begin{array}{c}\text { Conectores } \\
300 \times 300 \mathrm{~mm} \\
\text { cámaras de } \\
\text { aire de } 150 \times 160 \mathrm{~mm}\end{array}$ & $\begin{array}{l}\text { T. aire } \\
\text { T. muro } \\
\text { T. muro } \\
\text { T. aire } \\
\text { Flujo calor } \\
\text { C. Global real } \\
\text { C. T. muro } \\
\text { C. Global normalizado }\end{array}$ & $\begin{array}{l}\mathrm{T}_{\mathrm{e} 1} \\
\mathrm{~T}_{\mathrm{s} 1} \\
\mathrm{~T}_{\mathrm{s} 2} \\
\mathrm{~T}_{\mathrm{i} 2} \\
\varnothing \\
\mathrm{U}_{\mathrm{R}} \\
\mathrm{C}_{\mathrm{M}} \\
\mathrm{U}\end{array}$ & $\begin{array}{l}\circ \mathrm{C} \\
\circ \mathrm{C} \\
\circ \mathrm{C} \\
\circ \mathrm{C} \\
\mathrm{Kcal} / \mathrm{h} \cdot \mathrm{m}^{2} \\
\mathrm{Kcal} / \mathrm{h} \cdot \mathrm{m}^{2} \\
\mathrm{Kcal} / \mathrm{h} \cdot \mathrm{m}^{2} \\
\mathrm{Kcal} / \mathrm{h} \cdot \mathrm{m}^{2} \\
\end{array}$ & $\begin{array}{l}\circ \mathrm{C} \\
\circ \mathrm{C} \\
\circ \mathrm{C} \\
\end{array}$ & $\begin{array}{c}4.5 \\
5.2 \\
24.7 \\
25.0 \\
5.92 \\
0.289 \\
0.303 \\
0.286 \\
\end{array}$ & $\begin{array}{l}25.41 \\
25.7 \\
44.2 \\
44.4 \\
7.10 \\
0.374 \\
0.385 \\
0.358 \\
\end{array}$ \\
\hline $\begin{array}{c}\text { Conectores } \\
300 \times 300 \mathrm{~mm} \\
\text { cámaras de aire } \\
\text { de } 150 \times 160 \mathrm{~mm} \\
\text { en junta }\end{array}$ & $\begin{array}{l}\text { T. aire } \\
\text { T. muro } \\
\text { T. muro } \\
\text { T. aire } \\
\text { Flujo calor } \\
\text { C. Global real } \\
\text { C. T. muro } \\
\text { C. Global normalizado }\end{array}$ & $\begin{array}{l}\mathrm{T}_{\mathrm{e} 1} \\
\mathrm{~T}_{\mathrm{s} 1} \\
\mathrm{~T}_{\mathrm{s} 2} \\
\mathrm{~T}_{\mathrm{i} 2} \\
\varnothing \\
\mathrm{U}_{\mathrm{R}} \\
\mathrm{C}_{\mathrm{M}} \\
\mathrm{U}\end{array}$ & $\begin{array}{l}\circ \mathrm{C} \\
\circ \mathrm{C} \\
\circ \mathrm{C} \\
\circ \mathrm{C} \\
\mathrm{Kcal} / \mathrm{h} \cdot \mathrm{m}^{2} \\
\mathrm{Kcal} / \mathrm{h} \cdot \mathrm{m}^{2} \\
\mathrm{Kcal} / \mathrm{h} \cdot \mathrm{m}^{2} \\
\mathrm{Kcal} / \mathrm{h} \cdot \mathrm{m}^{2}\end{array}$ & $\begin{array}{l}\circ \mathrm{C} \\
\circ \mathrm{C} \\
\circ \mathrm{C}\end{array}$ & $\begin{array}{c}4.6 \\
5.3 \\
24.7 \\
25.0 \\
5.84 \\
0.287 \\
0.300 \\
0.284\end{array}$ & $\begin{array}{c}25.4 \\
25.6 \\
43.9 \\
44.4 \\
7.27 \\
0.383 \\
0.396 \\
0.368\end{array}$ \\
\hline
\end{tabular}

\section{TABLA 3}

\begin{tabular}{lll}
\hline TRADICIONAL & \\
\hline
\end{tabular}

Las condiciones de movimiento de aire se programaron para conseguir unos coeficientes de pelicula cercanos a 7 y $20 \mathrm{kcal} / \mathrm{h} . \mathrm{m}^{2}$ oC.

Las temperaturas se han controlado en las superficies del muro, asi como en el aire a unos $150 \mathrm{~mm}$ de las mismas, mediante termopares, siendo cada valor la media de cinco de éstos y el tiempo de muestreo de 20 segundos.

Los flujos se midieron en las diversas zonas indicadas mediante fluximetros de $300 \times 300 \times$ $8 \mathrm{~mm}$.

En las tablas 1 y 2 se muestran los resultados obtenidos.

Los valores $U$ teóricos calculados han sido: Plastbau l: 0,41 kcal/h.m² oC; Plastbau II: con huecos de aire: $0,28 \mathrm{kcal} / \mathrm{h} . \mathrm{m}^{2}$ oC; Plastbau II: con huecos rellenos de hormigón: 0,29 kcal/h.m ${ }^{2}$ oC, que se encuentran entre los experimentales obtenidos para las condiciones.climáticas de verano e invierno.

A la vista de los resultados puede observarse que la resistencia térmica de ambos sistemas es muy grande cumpliendo las exigencias de la norma NBE/CT, 79 en todas las zonas climáticas. 




Fig. 4.

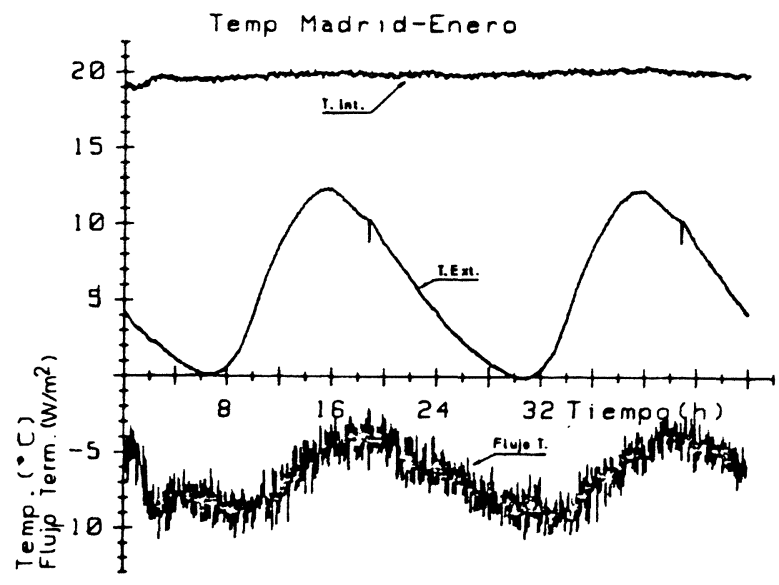

Fig. 5.

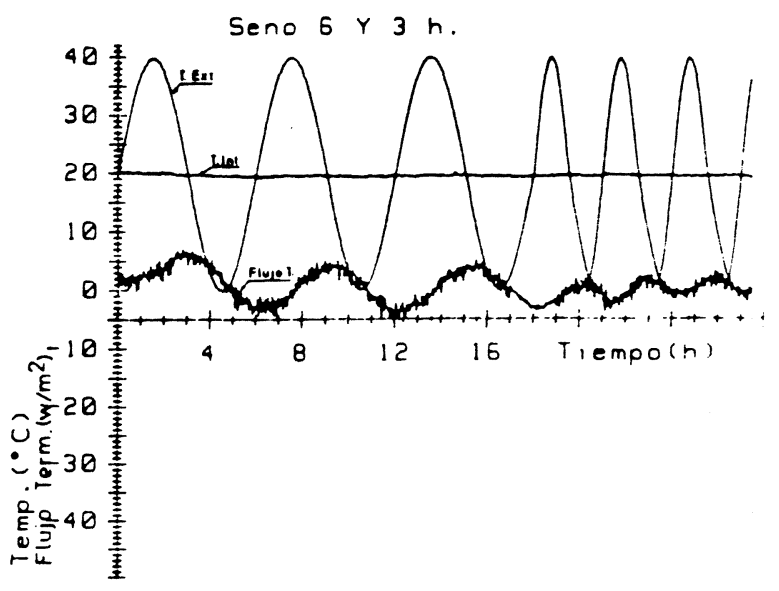

Fig. 6.

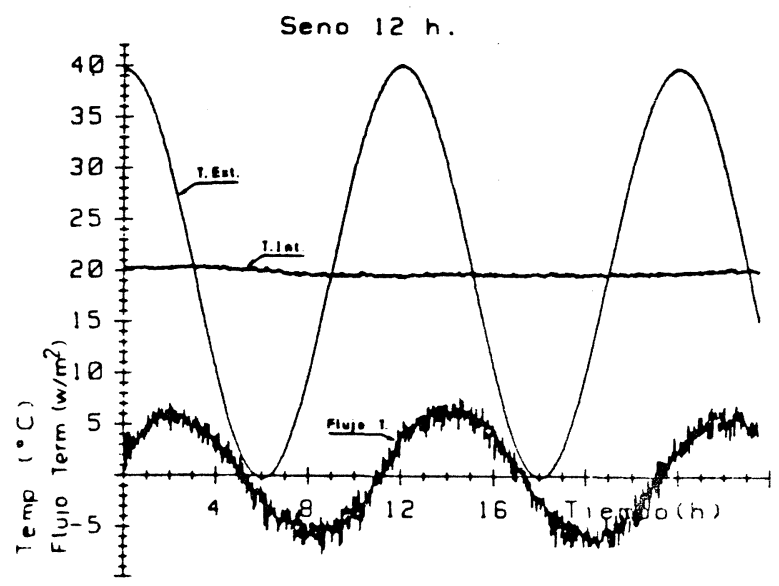

Fig. 7.

TABLA 4

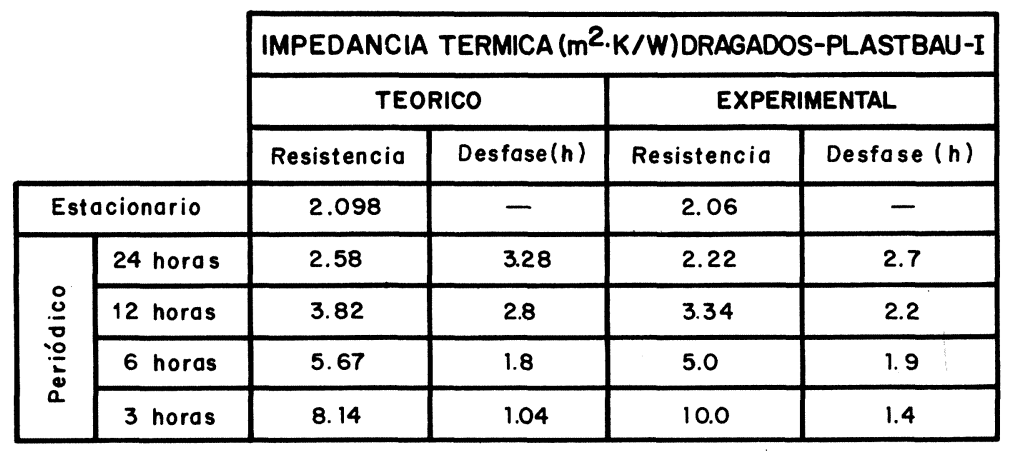

Se ha observado la escasa influencia de la variación de densidad de conectores, así como la existencia de juntas. No se observaron diferencias en el Plastbau II entre las zonas de huecos vacios y llenos de hormigón, justificable por la equivalencia entre las resistencias térmicas del hormigón y de la cámara de aire.

En la tabla 3 se comparan los resultados obtenidos con los correspondientes a dos cerramientos de uso frecuente.

\section{ENSAYOS EN REGIMEN VARIABLE}

Con el fin de conocer el comportamiento de estos muros en condiciones más próximas a la realidad se ha realizado un estudio teórico y experimental sobre el Sistema Dragados-Plastbau I.

Sobre el muro construido en el laboratorio y con el mismo dispositivo experimental descrito anteriormente se han reproducido en una de las caras la temperatura interior de $20 \circ \mathrm{C}$ y en la otra 
ondas sinusoidales entre $00 \mathrm{C}$ y $400 \mathrm{C}$ y periodos de 24, 12, 6 y 3 horas. Asimismo, se ha reproducido la onda de temperatura de un dia típico del mes de enero en Madrid.

En las figuras 4, 5, 6, 7 se muestran los resultados de los flujos obtenidos.

Puede observarse como ondas térmicas con la misma amplitud dan lugar a flujos térmicos diferentes, que disminuyen a medida que disminuye el periodo de la perturbación.

Si definimos la impedancia térmica como el cociente entre la amplitud de la onda de temperatura y la amplitud de la onda de flujo, se obtienen los valores que se indican en la tabla 4 . En ella puede comprobarse la mejora del comportamiento térmico del muro en régimen variable con relación al régimen estacionario.

En esta misma tabla también se indican el valor teórico y medio de los obtenidos por el método analítico (3) y por el método de impedancia térmica realizado por analogia eléctrica en ordenador (4).

En la actualidad se está estudiando el sistema D-P II en régimen variable y los resultados hasta ahora obtenidos conducen a que su comportamiento es superior al obtenido con el D-P I.

\section{CONCLUSIONES}

Las cargas térmicas reales, es decir en régimen variable, serán siempre inferiores a las deducidas del estudio en régimen estacionario, por lo que se recomienda este cálculo térmico, teniendo en cuenta las condiciones a las que vaya a estar sometido el cerramiento en cada aplicación.

\section{BIBLIOGRAFIA}

(1) CARRASCO, J. A.: «Aprovechamiento de la inercia térmica en el ahorro de energía". Tesis Doctoral, Instituto Quimico de Sarriá, Barcelona, 1983.

(2) CARRASCO, J. A.; GARCIA DE VINUESA, S.: "Determinación experimental del flujo térmico transmitido por conducción en régimen variable a través de cerramientos». Electrotermia 86, Zaragoza, nov. 1986.

(3) DIAZ, J. M.; DE ELVIRA, C.; ALVAREZ, I.; HERRERA, O.: "Método analitico para el estudio de la transmisión de calor por conducción en muros multicapa sometidos a variaciones periódicas de temperatura". Electrotermia 86, Zaragoza, nov. 1986.

(4) DOMINGUEZ, M.; CARRASCO, J. A.; ALVAREZ, I.; HERRERA, O.: "Estudio de la conducción de calor en muros multicapa en régimen periódico por analogía eléctrica con ordenador». Electrotermia 86, Zaragoza, nov. 1986.

\section{publicación del i.e.t.c.c.}

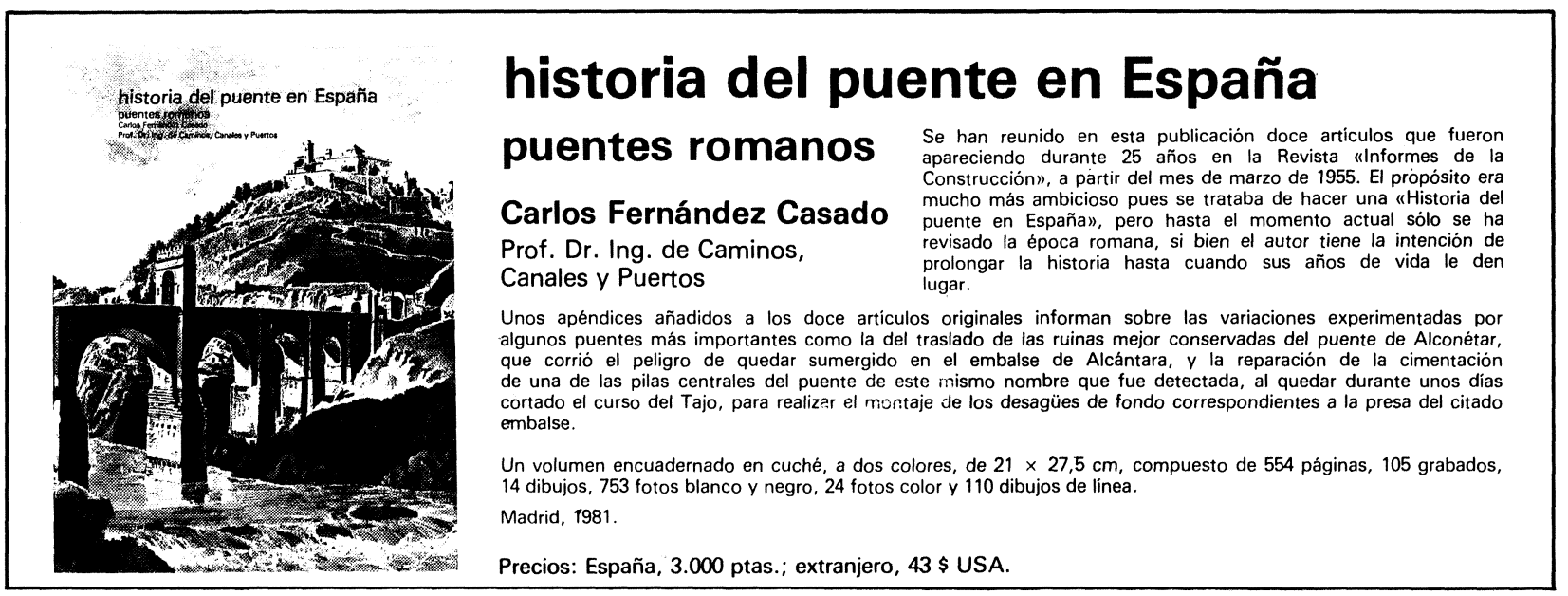

